\title{
Effects of Displayed Error Scaling in Compensatory Roll-Axis Tracking Tasks
}

\author{
S.W. Breur, ${ }^{*}$ D.M. Pool ${ }^{\dagger}$ M.M. van Paassen,${ }^{\ddagger}$ and M. Mulder ${ }^{\S}$ \\ Delft University of Technology, Delft, The Netherlands
}

\begin{abstract}
This paper describes an investigation into the effects of displayed error scaling on manual control behavior during compensatory roll-axis tracking. Previous experiments have indicated that for compensatory displays that, similar to an artificial horizon, present the roll tracking errors in rotational form, the deviations for typical quasi-random forcing function signals are comparatively small and difficult to perceive. This was found to lead to degraded tracking performance and lower crossover frequencies than would be expected. To investigate this, a roll-axis tracking experiment has been performed in which the scaling of the presented tracking errors was varied from 0.5 to 5 times the true tracking error. In addition, both double integrator dynamics and typical conventional roll dynamics of a small jet aircraft were considered in a mixed experimental design. The main hypothesis for this experiment was that increased scaling of the presented roll-axis tracking error would result in improved tracking performance and correlation of manual control inputs with the target forcing function signal. In addition, these effects were hypothesized to be more pronounced for the more difficult double integrator dynamics. For both controlled elements, both tracking performance and linearity of pilot control were indeed found to increase with increasing display gain, leveling of for the highest considered display gains. Further analysis of manual control behavior using McRuer et al.'s Precision Model revealed marked changes in the adopted control strategy due to changes in displayed error scaling, which were found to be highly similar for both controlled elements.
\end{abstract}

\section{Introduction}

Since the 1940s, a large research effort has been dedicated to the development of mathematical models capable of describing and predicting human manual control behavior. One of the more seminal works that resulted from this effort is that of McRuer et al., ${ }^{1}$ which introduced the Crossover and Precision Models that allow for the modeling of human manual control in compensatory tracking tasks. In addition, McRuer and $\mathrm{Jex}^{2}$ compiled an excellent overview of the factors that affect human behavior and performance during manual control, for instance making a distinction between the defining features of a control task and additional influences such as those imposed by the environment and operator-centered issues such as motivation.

Using the work of McRuer et al. as a starting point, many later experiments have been performed to investigate the effects of these different variables on manual control behavior. Notable examples are the work of Wasicko et al. ${ }^{3}$ that attempted to extend the principles and models introduced by McRuer et al. to pursuit tracking and the many investigations into the effects of physical motion feedback on pilot behavior. ${ }^{4-9}$ The modeling of pilot control behavior continues to receive considerable interest, due to its importance for, for example, the assessment of simulator fidelity ${ }^{10-12}$ and handling qualities evaluations. ${ }^{13,14}$

Recent investigations into compensatory roll attitude tracking performed at Delft University of Technology ${ }^{12,15}$ raised questions about the effects of the presentation of the roll tracking errors on the adopted manual control strategy. Both these experiments used a visual display similar to an artificial horizon for presenting the roll tracking error in the form of a rotation of the horizon line with respect to a fixed aircraft symbol. In addition, the typical quasi-random

\footnotetext{
*MSc. student, Control and Simulation Division, Faculty of Aerospace Engineering, P.O. Box 5058, 2600GB Delft, The Netherlands; s.w.breur@student.tudelft.nl

${ }^{\dagger}$ PhD candidate, Control and Simulation Division, Faculty of Aerospace Engineering, P.O. Box 5058, 2600GB Delft, The Netherlands; d.m.pool@tudelft.nl. Member AIAA.

${ }^{\ddagger}$ Associate Professor, Control and Simulation Division, Faculty of Aerospace Engineering, P.O. Box 5058, 2600GB Delft, The Netherlands; m.m.vanpaassen@tudelft.nl. Member AIAA.

${ }^{\S}$ Professor, Control and Simulation Division, Faculty of Aerospace Engineering, P.O. Box 5058, 2600GB Delft, The Netherlands; m.mulder@tudelft.nl. Member AIAA.
} 
forcing function signals adopted in these experiments induced relatively small tracking errors (max. $5 \mathrm{deg}$ ). This combination of display and forcing function signal is suspected to have led to difficulties in the perception of the tracking errors during these experiments, resulting in reduced correlation of control inputs with the applied forcing function and reduced linearity of manual control behavior. The comparatively poor tracking of the forcing function signals and low crossover frequencies reported for these studies were thought to be further testament to this fact.

From these previous observations, it was hypothesized that an increase in the magnitude of the tracking errors presented on the compensatory displays in these experiments would have resulted in improved tracking performance and higher linearity of manual control behavior. This increased presented tracking error magnitude can be achieved in two ways: by increasing the amplitude of the applied forcing function signals or by scaling of the tracking errors presented on the compensatory display. As the problem was found to mainly result from the adopted display format and to avoid excessively high-magnitude forcing function signals, it was decided to investigate this problem in terms of the magnitude and scaling of the presented tracking errors. Previous research into the effects of display quality on manual control behavior has been performed, ${ }^{16}$ but the effects of scaling of presented tracking errors have never been quantified explicitly.

This paper describes the results of an experiment that has been performed at Delft University of Technology to investigate the effect of the displayed error scaling on manual control behavior during a roll-axis target-following task. The experiment was designed to analyze the effects of displayed error scaling on tracking performance and control activity, as well as pilot-vehicle system crossover frequency, phase margin, effective time delay and linearity of pilot control behavior. A slightly simplified version of McRuer et al.'s ${ }^{1}$ Precision Model was fit onto the measurement data to quantify the influence of displayed error scaling on manual control behavior. Finally, as the effects of display scaling were anticipated to depend on the the dynamics of the controlled element, this interaction was explored through the use of two controlled elements, one representative for the aileron-to-roll dynamics of a small jet aircraft and a pure double integrator system.

First, this paper gives a brief overview of the compensatory roll-axis target-following task that is under investigation and the most important literature available for modeling manual control in such a control task. Then, Section III provides the full details of the experiment. The experimental results are presented in Section IV. The paper ends with a discussion of the main experimental results and conclusions.

\section{Modeling Manual Control Behavior During Tracking}

\section{II.A. Compensatory Target-Following}

As indicated in Fig. 1, a target-following task is a tracking task in which a human operator is required to make the controlled variable, in this case the roll angle $\phi$, follow a predefined reference trajectory represented by the target forcing function $f_{t}$. The human operator, whose dynamics are indicated by the symbol $Y_{p}$, gives control inputs $u$ to the controlled element dynamics $Y_{c}$ to achieve this. During compensatory tracking the human operator only reacts to tracking error $e$, that is, the difference between $f_{t}$ and $\phi$. In most research into human dynamics during compensatory control a quasi-random multisine signal has been used as the target forcing function $f_{t},{ }^{1,2,17}$ while other types of signals, such as ramps ${ }^{12,18}$ and steps ${ }^{19}$ have also been used occasionally. This paper considers a compensatory targetfollowing task where $f_{t}$ is a typical quasi-random multisine signal, as also used in previous experiments. ${ }^{12,18,20,21}$

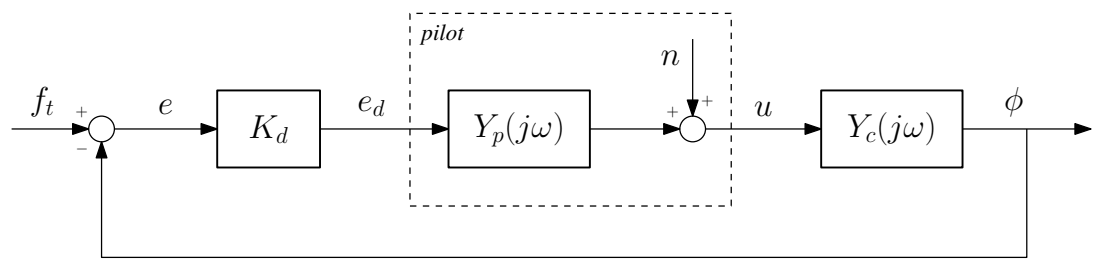

Figure 1. Target-following task with quasi-linear human controller model.

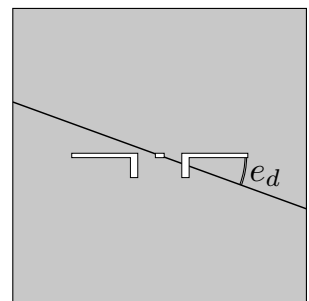

Figure 2. Compensatory display.

The focus of the research presented here is on the presentation of the tracking error on a compensatory visual display that represents a simplified artificial horizon image, as depicted in Fig. 2. As also shown in Fig. 1, the experiment described in this paper evaluates the effects of scaling the displayed tracking error $e_{d}$ by an error scaling gain $K_{d}$ (that is, $e_{d}=K_{d} e$ ) on manual control behavior and tracking performance. Displays similar to the one depicted in Fig. 2 have been used in previous experiments that investigated roll attitude tracking, ${ }^{6,7,12,15}$ mostly for the 
case where the actual tracking error was depicted on the compensatory display $\left(K_{d}=1\right)$. For the experiment described in Ref. 15, degraded manual tracking performance has been reported and attributed to the fact that the tracking errors, which were typically no larger than $5 \mathrm{deg}$, were difficult to perceive from such a display format.

\section{II.B. McRuer's Crossover Model and Precision Model}

McRuer and $\mathrm{Jex}^{2}$ have proposed to model the dynamics of human operators during tracking as a quasi-linear system, consisting of a linear response function $Y_{p}$ that relates perceived tracking errors to control inputs $u$ and a nonlinear remnant signal $n$ to account for all control inputs that are uncorrelated with the target signal $f_{t}$. During their experiments, McRuer et al. ${ }^{1}$ found that the open-loop response, that is, the dynamics of the combined pilot-vehicle system, showed constant behavior over a wide range of controlled elements dynamics, due to the adaptability of the human controller. Controllers showed integrating, proportional or differentiating control behavior depending on the controlled element dynamics $Y_{c}$, in order to yield an open-loop pilot-vehicle system with pure integrator-like dynamics in the crossover region, while retaining sufficient closed-loop stability margins. ${ }^{2}$ This resulted in the formulation of the Crossover Model as defined by Eq. (1), which describes the open-loop response of the pilot-vehicle system $Y_{O L}$ in the crossover region.

$$
Y_{O L}(j \omega)=Y_{p}(j \omega) Y_{c}(j \omega) \doteq \frac{\omega_{c}}{j \omega} e^{-j \omega \tau_{e}}
$$

The Crossover Model parameters as indicated in Eq. (1) are the effective time delay $\tau_{e}$ and the unity-gain crossover frequency $\omega_{c}$. This open-loop system response as defined by Eq. (1) can be decomposed into the known controlled element dynamics $Y_{c}$ and a human operator model $Y_{p}$, thereby showing the human operator's adaptation to the dynamics characteristics of $Y_{c}$. The most extensive human operator model proposed by McRuer et al. ${ }^{1}$ is the Precision Model, which accounts for this adaptation of the human operator dynamics, as well as the effects of manipulator and neuromuscular system dynamics:

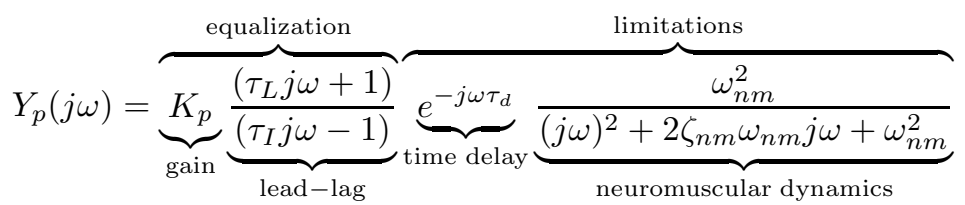

In Eq. (2), $K_{p}$ is the pilot gain which forms the equalization part of the Precision Model, together with the lead time constant $\tau_{L}$ and lag time constant $\tau_{I}$. The other half of the Precision Model accounts for controller limitations, that is, a pilot time delay $\tau_{d}$ and the dynamics of the neuromuscular actuation required for giving a control input. The combined dynamics of the human neuromuscular system and the manipulator are modeled by a second-order mass-spring-damper system with the neuromuscular bandwidth $\omega_{n m}$ and damping coefficient $\zeta_{n m}$ as parameters. ${ }^{21}$ Note that the low-frequency component of the neuromuscular system as included in the original Precision Model of McRuer et al. ${ }^{1}$ has been omitted here.

\section{II.C. Variables that Affect Manual Control Behavior}

The control behavior that is adopted during a manual control task, and hence the attained level of task performance, is heavily dependent on the specifics of the control task itself and the conditions under which it is performed. As depicted in Fig. 3, McRuer and Jex ${ }^{2}$ defined four categories of key variables, with the most notable examples of each, that affect a pilot-vehicle system:

- Task variables: these comprise the system inputs and control system elements external to the pilot that have a direct and explicit effect on the control task. McRuer and Jex ${ }^{2}$ identified four factors among these to be the dominant factors to affect pilot dynamics: the forcing function signal(s), the displays, the manipulator, and the controlled element dynamics.

- Environmental variables: these are the factors external to the pilot that are not associated with the control task itself, but rather with the environment in which the control task is performed. Examples are whether the experiment is conducted in a fixed-base simulator, moving-base simulator or in-flight, as well as factors such as g-loading imposed on the pilot, temperature, vibrations, and the ambient illumination.

- Operator-centered variables: variables internal to the operator that affect his control behavior, such as fatigue, motivation, stress, and amount of previously performed training. 
- Procedural variables: these are the factors that depend on the manner in which experimental measurements of control behavior are collected. Examples are operator briefing and instructions, amount of practice, and the order of presentation of different experimental conditions.

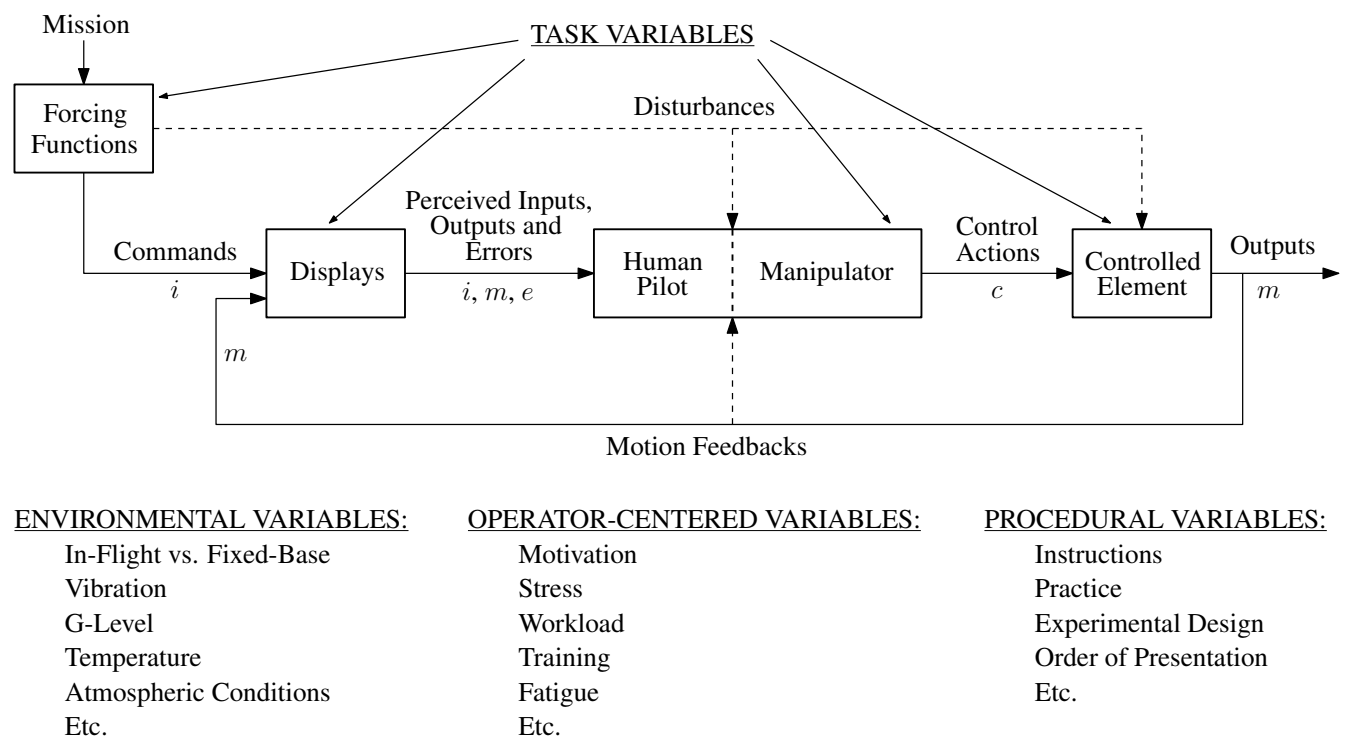

Figure 3. Variables that affect a pilot-vehicle system as defined by McRuer and Jex. ${ }^{2}$

In general, experiments in which measurements of human manual control behavior are collected are designed to keep procedural and operator-centered variables as constant as possible, to limit their influence on the results. Some investigations have been made of the explicit influence of some of the environmental variables on manual control. ${ }^{22-24}$ Evaluating the effects of variations in the task variables as indicated in Fig. 3, however, makes up the large majority of the research into manual control during tracking. Considerable research has been performed on the effects of motion feedback on pilot dynamics, ${ }^{6,7,15}$ while variations in forcing functions and controlled element dynamics have also been the focus of a large number of experiments. ${ }^{1,2,17}$ Several investigations have also been made into the effects of changing the various aspects of visual displays. For example, experiments were done on the effects of display format ${ }^{25}$ as well as the effects of reduced display quality. ${ }^{16}$ However, no prior research has been performed to explicitly investigate the effects of scaling the presented tracking error on pilot dynamics during compensatory tracking.

\section{Experiment}

\section{III.A. Method}

\section{III.A.1. Experiment Setup}

To investigate the effect of scaling tracking errors on a compensatory display, an experiment was performed in the fixed-base simulator in the Human-Machine Systems laboratory of the Faculty of Aerospace Engineering at Delft University of Technology, see Fig. 4. Participants performed a roll-axis target-following task using the compensatory display shown in Fig. 2, which was presented on the primary flight display of the simulator. The update rate of the primary flight display was $60 \mathrm{~Hz}$ and the time delay associated with presenting images on this display (including the projection) was measured to be around $20 \mathrm{~ms}$ using a custom delay measurement system. ${ }^{26}$

Control inputs $u$ were given using the roll axis of an electro-hydraulic, servocontrolled, passive sidestick, which is also depicted in Fig. 4. The maximum roll deflection of the sidestick were set to \pm 15 deg and rotational stick stiffness was

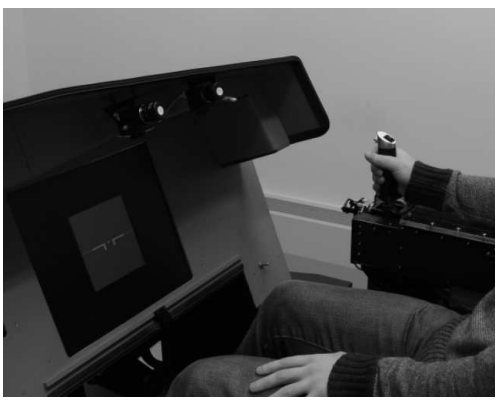

Figure 4. Experiment setup. $0.087 \mathrm{Nm} / \mathrm{deg}$ over the full deflection range. The sidestick had no breakout in roll. The pitch axis of the stick was kept fixed in the neutral position during the experiment. 


\section{III.A.2. Forcing Function}

A single tracking run was defined to last 100 seconds, with a measurement time $T_{m}$ equal to 81.92 seconds. The first 18.08 seconds of each run were considered the run-in time, which participants could use to reach a stable control strategy. The data from these first 18.08 seconds were not considered for analysis of control behavior. The target forcing function $f_{t}$ used for the experiment was a multisine signal built up from ten sinusoids according to:

$$
f_{t}(t)=\sum_{i=1}^{10} A_{f}\left(\omega_{i}\right) \sin \left(\omega_{i} t+\phi_{f}\left(\omega_{i}\right)\right)
$$

In Eq. (3), $\omega_{i}$ represents the frequency of these sinusoids, which were selected to all be integer multiples of the experiment measurement time base frequency $\omega_{m}=2 \pi / T_{m}=0.0767 \mathrm{rad} / \mathrm{s}$, according to $\omega_{i}=n_{i} \omega_{m}$. A secondorder low-pass filter was used to compute the amplitude distribution $A_{f}\left(\omega_{i}\right)$ of these sinusoids, as defined by Eq. (4):

$$
A_{f}\left(\omega_{i}\right)=K_{f}\left|\frac{\left(T_{A_{1}} j \omega_{i}+1\right)^{2}}{\left(T_{A_{2}} j \omega_{i}+1\right)^{2}}\right|
$$

The amplitude distribution given by Eq. (4) is the same as the one used for many previous experiments. ${ }^{20,21}$ Here, $K_{f}=2.133, T_{A_{1}}=0.1 \mathrm{~s}$ and $T_{A_{2}}=0.8 \mathrm{~s}$ were used to yield a signal with time-domain variance of $3 \mathrm{deg}^{2}$. The sinusoid phase distribution $\phi_{f}\left(\omega_{i}\right)$ was chosen to produce a forcing function with a Gaussian distribution. ${ }^{14}$ The numerical values of the sinusoid amplitudes, frequencies, and phases that were used for generating $f_{t}$ can be found in Table 1. The time trace of the target signal is shown in Fig. 5.

\begin{tabular}{|c|c|c|c|c|}
\hline$i$ & $n_{i}$ & $\omega_{i}$ & $A_{f}$ & $\phi_{f}$ \\
\hline- & - & $\mathrm{rad} / \mathrm{s}$ & deg & $\mathrm{rad}$ \\
\hline 1 & 6 & 0.460 & 1.8827 & 0.5525 \\
\hline 2 & 13 & 0.997 & 1.3167 & 4.6607 \\
\hline 3 & 27 & 2.071 & 0.5941 & 3.8287 \\
\hline 4 & 41 & 3.145 & 0.3199 & 6.2324 \\
\hline 5 & 53 & 4.065 & 0.2147 & 3.1959 \\
\hline 6 & 73 & 5.599 & 0.1330 & 5.1069 \\
\hline 7 & 103 & 7.900 & 0.0846 & 1.7919 \\
\hline 8 & 139 & 10.661 & 0.0618 & 0.7950 \\
\hline 9 & 194 & 14.880 & 0.0480 & 3.0965 \\
\hline 10 & 229 & 17.564 & 0.0439 & 3.6026 \\
\hline
\end{tabular}

Table 1. Multisine properties of $f_{t}$.

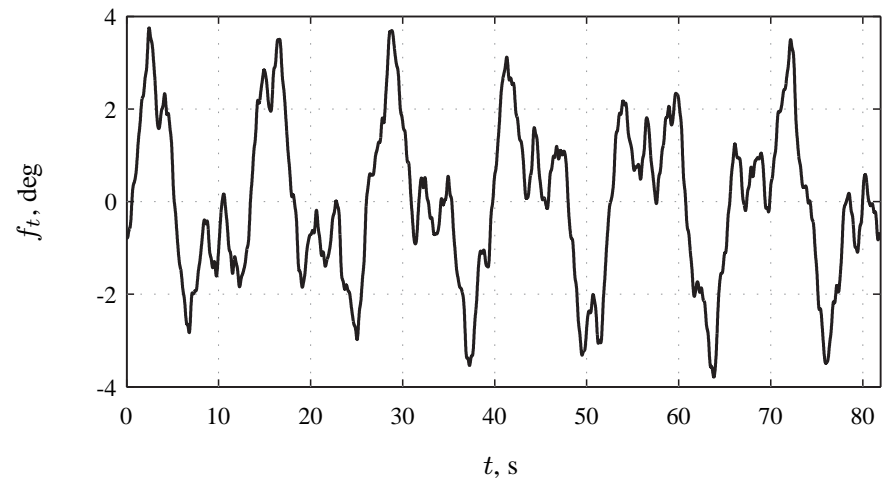

Figure 5. Time trace of the target forcing function $f_{t}$.

\section{III.A.3. Controlled Element Dynamics}

The dynamics of the controlled element, $Y_{c}$, have been shown to have a marked effect on manual control behavior during tracking in numerous occasions. ${ }^{1,2,14}$ Therefore, it is likely that the effect of scaling the presented tracking error as investigated in this paper, will also depend on the dynamics of $Y_{c}$. To tie in with the results from seminal work on modeling of manual control during tracking ${ }^{1,2}$ and more recent experiments into manual aircraft control tasks, ${ }^{12,18}$ it was decided to evaluate the effect of $K_{d}$ (see Fig. 1) for two different controlled elements.

The first controlled element represented the aileron-to-roll dynamics of a Cessna Citation I aircraft with yaw damper on, linearized at an altitude of $10,000 \mathrm{ft}$ and airspeed of $160 \mathrm{kt}$ :

$$
Y_{c, c i t}(s)=-11.95 \frac{(s+0.2928)\left(s^{2}+3.377 s+3.829\right)}{(s+2.153)(s+0.3894)(s-0.01109)\left(s^{2}+3.216 s+4.470\right)}
$$

The frequency response of this controlled element is depicted in Fig. 6 . In addition, a vertical dashed indicates the break frequency that can be defined for this controlled element, $\omega_{b}=2.153 \mathrm{rad} / \mathrm{s}$, which represents the characteristic frequency of the roll subsidence mode. Note from Fig. 6 that $Y_{c, c i t}$ is approximately $K / s$ for frequencies below $\omega_{b}$ and approximately $K / s^{2}$ above $\omega_{b}$.

The second controlled element considered in the experiment is a pure double integrator, which was selected to coincide with the dynamics of $Y_{c, c i t}$ at high frequencies (see also Fig. 6): 
(a) Magnitude

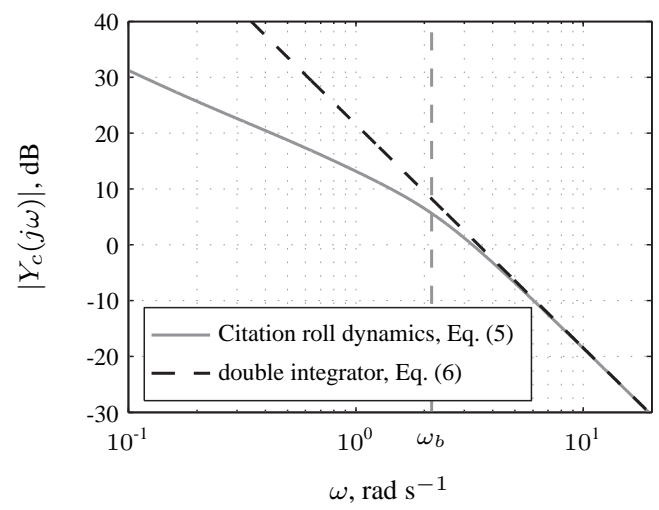

(b) Phase

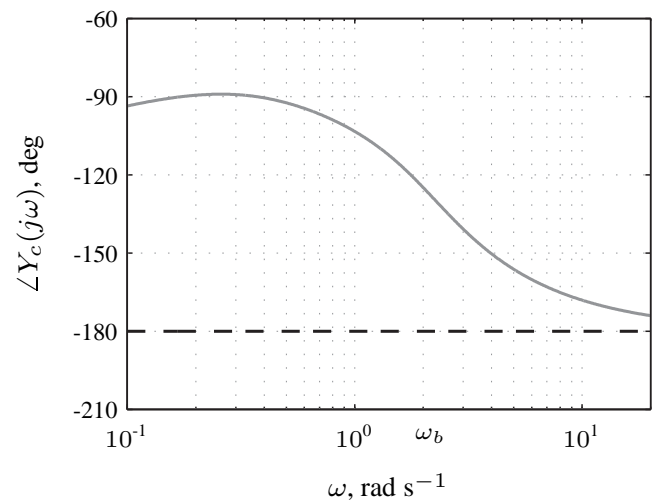

Figure 6. Frequency response of both controlled element dynamics.

$$
Y_{c, d i}(s)=\frac{-11.95}{s^{2}}
$$

As Fig. 6 shows, this system has significantly less phase lead in the crossover region than the Citation roll dynamics. This yields a markedly less stable system that requires human operators to perform more lead equalization - that is, starting at lower frequencies - than they would for $Y_{c, c i t}{ }^{1,2}$

\section{III.A.4. Independent Variables}

Two different independent variables were considered in the experiment. The main goal of this experiment was the evaluation of the displayed error scaling $K_{d}$ on manual control behavior and tracking performance. To this end, five different levels of displayed error scaling gain were tested in the experiment: $K_{d}=0.5,1.0,2.0,3.0$ and 5.0. In addition, the effect of $K_{d}$ was evaluated for both controlled elements introduced in Sec. III.A.3 according to a factorial experiment design.

\section{III.A.5. Dependent Measures}

For each experimental measurement run the time traces of three signals were recorded: the system roll angle $\phi$, the tracking error $e$, and the control input $u$. From these signals, the mean variance of the error $\sigma_{e}^{2}$ and mean variance of the control input $\sigma_{u}^{2}$ were computed as measures of tracking performance and control activity, respectively.

From the recorded tracking error and control input signals of each run a frequency-domain describing function of the pilot dynamics was estimated using the Fourier coefficients (FC) method. ${ }^{4,27}$ Using interpolation on this estimated describing function, the pilot-vehicle system crossover frequency $\omega_{c}$ and phase margin $\varphi_{m}$ were computed for each individual run, after which the effective time delay $\tau_{e}$ was calculated under the assumption that the Crossover Model was valid for these measurements: $\tau_{e}=\left(\pi / 2-\varphi_{m}\right) / \omega_{c}{ }^{2}$ Additionally, the correlation coefficient $\rho^{2}$ was computed to assess the correlation of control inputs with the target signal $f_{t} \cdot{ }^{1}$

Finally, McRuer's Precision Model was fit to the measured data using the time-domain maximum likelihood estimation procedure detailed in Ref. 20. Note that due to the fact that for both controlled elements introduced in Sec. III.A.3 lead equalization needs to be performed, the lag term shown in Eq. (2) was omitted here, reducing the equalization characteristic to a pure lead. Changes in the parameters of the resulting model - the pilot gain $K_{p}$, the pilot time delay $\tau_{d}$, the lead time constant $\tau_{L}$, the neuromuscular bandwidth $\omega_{n m}$ and the neuromuscular damping coefficient $\zeta_{n m}$ - were then analyzed to reveal underlying changes in manual control behavior due to the manipulation of the independent variables of the experiment.

\section{III.A.6. Experimental Design, Subjects, and Procedures}

The two independent variables of the experiment were evaluated in a mixed experimental design. The displayed error gain $K_{d}$ was considered as a within-subjects factor, that is, all subjects performed the target following task for all values of $K_{d}$. The controlled element dynamics were considered as a between-subjects variable in the experiment, to limit the amount of time needed for each subject to complete the experiment. Each participant was randomly assigned to either 
one of the controlled elements. To minimize the influence of learning effects and fatigue on measurement results, a balanced Latin square was used to determine the order in which each subject performed the different displayed error scale settings.

Ten male subjects took part in the experiment. All participants were students and personnel from the Control and Simulation Division, who has shown to be highly capable and consistent in performing the tracking task considered in the experiment. Furthermore, the majority of the participants had extensive experience from previous laboratory tracking experiments.

Collecting the measurements for each subject typically took half a day, with participants starting with some test runs to familiarize themselves with the display and controlled element dynamics, and then proceeding to the actual experiment. Each displayed error scaling setting was then tested in the order determined by the balanced Latin square. Training for each condition was done by letting participants perform as many runs as needed to reach a stable level of performance. Once stable performance was achieved, five repetitions were taken as the measurement data. Regular breaks were taken to limit fatigue and subjects were informed about their performance after each run.

\section{III.B. Hypotheses}

Three hypotheses were formulated in advance of this experiment:

1. Increasing the displayed error gain $K_{d}$ will yield an increase in the correlation of control behavior with the forcing function signal and an increase in tracking performance. With tracking errors being more easily to perceive, especially those induced by the comparatively low-magnitude high-frequency sinusoids in the forcing function signal, participants are expected to adapt their control strategy for optimal performance.

2. This increase in tracking performance will be achieved by an increase in the crossover frequency $\omega_{c}$. This increase in crossover frequency, which is to some extent equivalent to the gain of pilot control, will most likely also be observable from an increase in control activity.

3. Due to the requirement for more lead equalization and the more difficult nature of target-following tasks performed with double integrator controlled element dynamics, ${ }^{1,2}$ it is expected that the observed effects of $K_{d}$ will be markedly larger in magnitude for the double integrator than for the Citation roll dynamics.

\section{Results}

The average values for the dependent variables presented in this section were found by averaging the results for each condition over the different participants and the five repetitions performed by each participant. Observed trends were tested for significance using Analysis of Variance (ANOVA). The effects of the within-subjects factor $K_{d}$ were analyzed using a repeated-measures ANOVA, for each controlled element separately. Mixed-design ANOVAs were used to check for further interactions between the displayed error scaling and the dynamics of the controlled element. All results from these ANOVAs for all considered dependent measures are listed in Table 2. If the assumption of sphericity was not met for any of the dependent variables, the Greenhouse-Geisser sphericity correction was applied. In Table 2 these occurrences are indicated with a superscript "gg".

\section{IV.A. Tracking Performance and Control Activity}

Fig. 7 shows the mean variance of the tracking error and control input signals as a function of displayed error scaling for both the Citation roll and double integrator controlled element dynamics. These quantities are measures of tracking performance and control activity, respectively. For both error and control signal variances, the contributions of the target forcing function and the remnant $n$ are depicted separately. ${ }^{5}$

\section{IV.A.1. Tracking Performance}

As can be seen in Fig. 7(a) and (b), the mean variance of the error was found to decrease with increasing display gain $K_{d}$ for both controlled elements. The sole exception to this trend is the error variance for $K_{d}=5.0$ and the Citation roll dynamics, which was found to be slightly higher than $\sigma_{e}^{2}$ for $K_{d}=3.0$. The performance increase (decrease in

$\sigma_{e}^{2}$ ) is the most pronounced for the lower values of $K_{d}$, whereas $\sigma_{e}^{2}$ appears to approach a constant value for the higher scaling gains. 
(a) Error variance, Citation roll dynamics

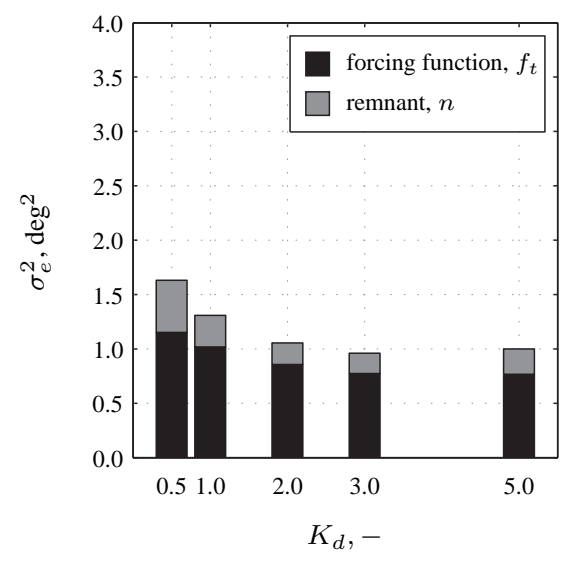

(c) Control variance, Citation roll dynamics

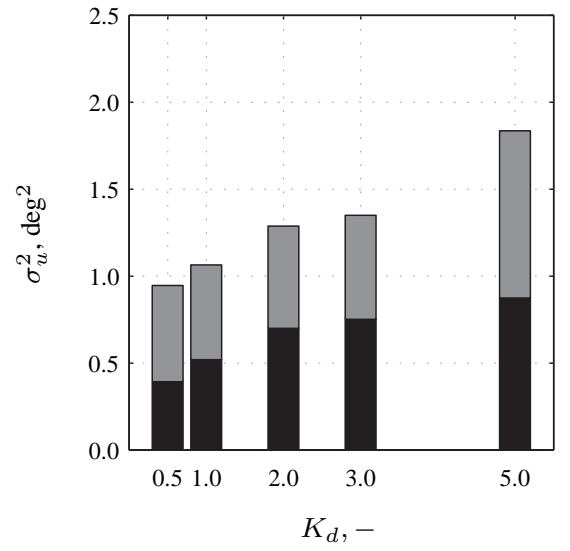

(b) Error variance, double integrator dynamics

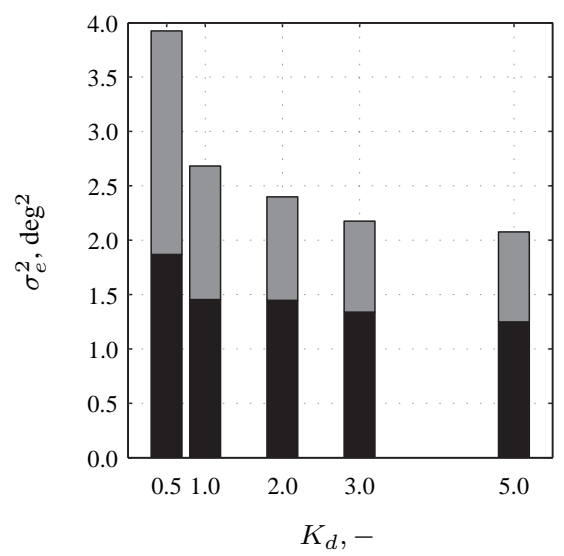

(d) Control variance, double integrator dynamics

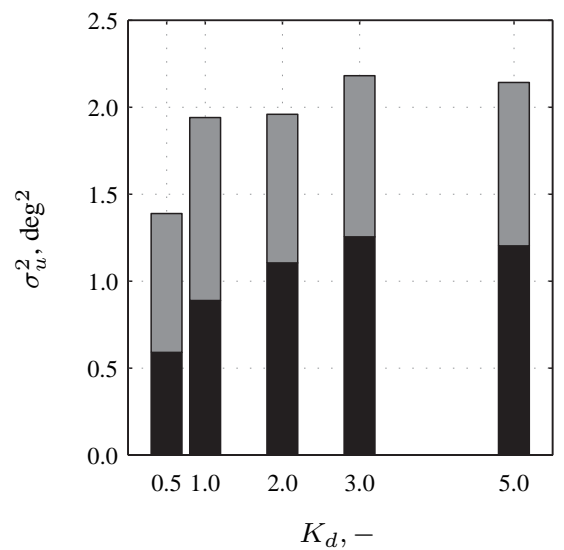

Figure 7. Mean variance of the error and control activity as function of $K_{d}$.

Table 2. ANOVA results.

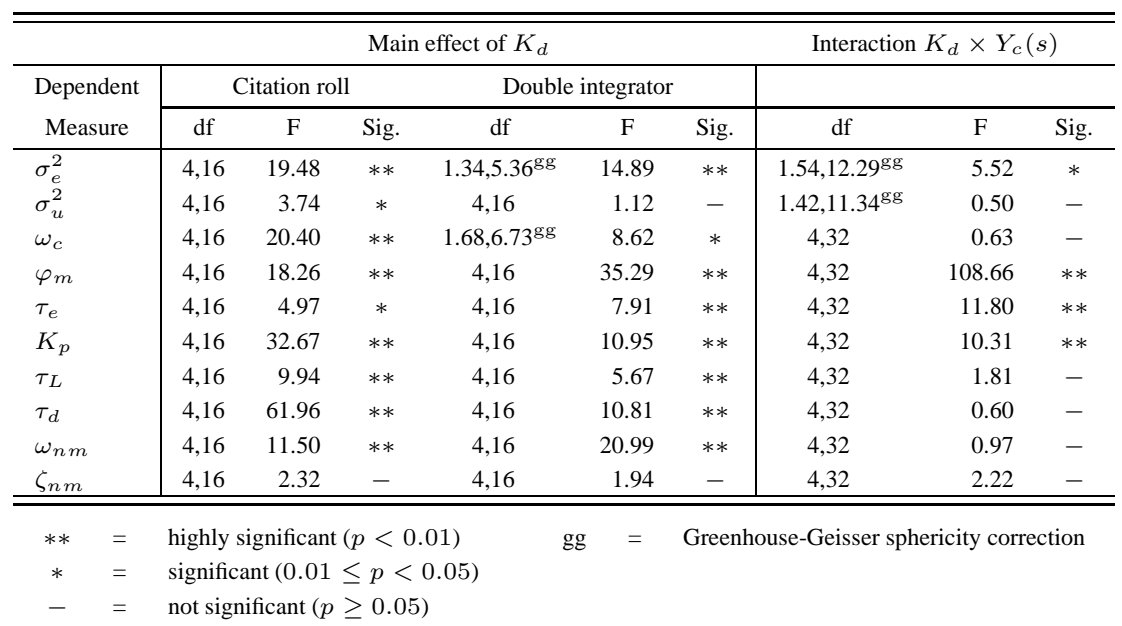


As can be verified from Table $2, K_{d}$ was found to have a highly significant effect on the mean variance of the error for the Citation roll dynamics, $F(4,16)=19.48, p<0.01$. For the double integrator dynamics, Mauchly's test indicated a violation of the sphericity assumption, $\chi^{2}(9)=24.29, p<0.05$. Correcting degrees of freedom using Greenhouse-Geisser estimates of sphericity $(\epsilon=0.335)$ indicated, however, that the displayed error scaling still had a highly significant effect on the mean variance of the error when using double integrator dynamics, $F(1.3,5.4)=$ $14.90, p<0.01$. These results are in line with the first hypothesis formulated for the experiment.

As Fig. 7(a) and (b) show, there is a considerable difference in tracking performance between the two controlled elements, with $\sigma_{e}^{2}$ being more than twice as large for most conditions for control of double integrator dynamics. This illustrates the difference in difficulty between the two systems. Furthermore, the reduction in $\sigma_{e}^{2}$ with increasing $K_{d}$ is more pronounced for the double integrator dynamics, which suggests the presence of an interaction between the effects of $K_{d}$ and $Y_{c}$ on the tracking error variance. While the sphericity assumption was violated for testing this interaction, $\chi^{2}(9)=21.432, p<0.05$, the use of Greenhouse-Geisser estimates of sphericity $(\epsilon=0.384)$ confirmed the presence of a significant interaction between displayed error scaling and controlled element dynamics, $F(1.5,12.3)=5.52$, $p<0.05$. This more marked effect of $K_{d}$ for the double integrator dynamics can be explained by the reduced stability of that system, which requires more human operators to generate more phase lead, which in turn is difficult for low values of $K_{d}$, due to the reduced perception of $e$.

The decomposition of the mean variance of the error reveals that the contribution of the target signal to $\sigma_{e}^{2}$ is typically larger than the contribution of the remnant $n$. Fig. 7 also shows that a greater portion of $\sigma_{e}^{2}$ results from the remnant for control of the double integrator dynamics than found for the Citation roll dynamics. For the Citation roll dynamics (Fig. 7(a)), an increase in error scaling is seen to result in a reduction in both the contributions of $f_{t}$ and $n$. For the double integrator dynamics, a similar trend was found, with both contributions to the total tracking error variance decreasing with increasing $K_{d}$. From Figs. 7(a) and (b), the main reduction in $\sigma_{e}^{2}$ appears to occur for $K_{d}=0.5$ to 2.0, where the reduction in the remnant component makes a dominant contribution to the total reduction in tracking error variance. The small observed improvement in performance for higher levels of scaling, on the other hand, shows comparable reductions in both the contributions of $f_{t}$ and $n$ to the total variance of the error.

\section{IV.A.2. Control Activity}

Fig. 7(c) shows a steadily increasing control input variance with increasing $K_{d}$ for the Citation roll dynamics, which was found to be a significant effect, $F(4,16)=3.74, p<0.05$. For the double integrator dynamics (see Fig. $7 \mathrm{~d}$ ), such a similarly clear trend is not found, with $\sigma_{u}^{2}$ remaining nearly constant for display gain values of 1.0 and higher. As a result, no significant effect of $K_{d}$ on $\sigma_{u}^{2}$ was found, $F(4,16)=1.12, p>0.05$. Still, the increasing trends in control variance depicted in Fig. 7 with increasing $K_{d}$ are in accordance with the second hypothesis that was formulated before the experiment, and suggest increased control gain for higher display gain values.

Comparing the measured control activity for the two controlled elements reveals considerable differences for displayed error scaling levels of 3.0 and below, with control activity levels for the Citation roll dynamics well below those found for the double integrator dynamics. However, control activity at $K_{d}=5.0$ was found to be at similar levels for both controlled elements, mainly due to a sharp increase in control activity for the Citation roll dynamics between display scaling gains of 3.0 and 5.0. While testing for an interaction between displayed error scaling and the dynamics of $Y_{c}$, Mauchly's test indicated a sphericity assumption violation, $\chi^{2}(9)=27.065, p<0.05$. Correcting for this with the use of Greenhouse-Geisser sphericity assumption $(\epsilon=0.354)$ revealed that no significant interaction took place between displayed error scaling and controlled element dynamics, $F(1.4,11.3)=0.50, p>0.05$.

As the decomposition in Fig. 7(c) and (d) shows, linear contributions and remnant contributions were of highly comparable magnitude for the control input variance. For the Citation roll dynamics, the increase in control input between $K_{d}=0.5$ and 3.0 can mostly be ascribed to an increase in the linear contribution of $f_{t}$, whereas an increase in remnant appears to be the main cause of the sharp increase in control activity between $K_{d}=3.0$ and 5.0. For the double integrator dynamics the increase in control activity was mostly caused by increases in contributions at the forcing function frequencies, with the remnant contribution remaining more or less constant.

\section{IV.B. Crossover Frequency, Effective Time Delay, and Phase Margin}

\section{IV.B.1. Crossover Frequency}

Fig. 8(a) shows the crossover frequency $\omega_{c}$ as a function of the display gain $K_{d}$. Variance bars indicate $95 \%$ confidence intervals. Note that the presented data for both controlled elements has been corrected for between-subject variance. Fig. 8(a) indicates that the crossover frequency was found to increase with increasing $K_{d}$, for both controlled element 
dynamics. Verification using an ANOVA showed this increase to be highly significant for the Citation roll dynamics, $F(4,16)=20.40, p<0.01$. For the integrator system dynamics, Mauchly's test indicated a violation of the sphericity assumption, $\chi^{2}(9)=20.065, p<0.05$. After adjustments using the Greenhouse-Geisser sphericity assumption $(\epsilon=0.420)$, the displayed error scaling was also found to have a significant effect on the crossover frequency for $Y_{c, d i}, F(1.68,6.73)=8.62, p<0.05$. This increase in crossover frequency with increasing $K_{d}$, which is in line with the second hypothesis formulated for the experiment, mainly occured for the lower displayed error scaling levels. Similar to the trends observed in tracking performance and control activity (Fig. 7), measured crossover frequencies are found to approach an approximately constant value for the highest considered values of $K_{d}$. As the trends in $\omega_{c}$ are highly similar for both controlled elements, an interaction between displayed error scaling and $Y_{c}$ appears to be nonexistent, an observation that is confirmed from the ANOVA results shown in Table 2: $F(4,32)=0.63, p>0.05$.

(a) Crossover frequency

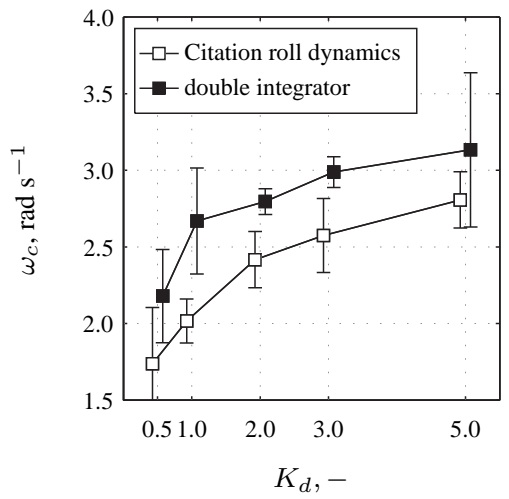

(b) Phase margin

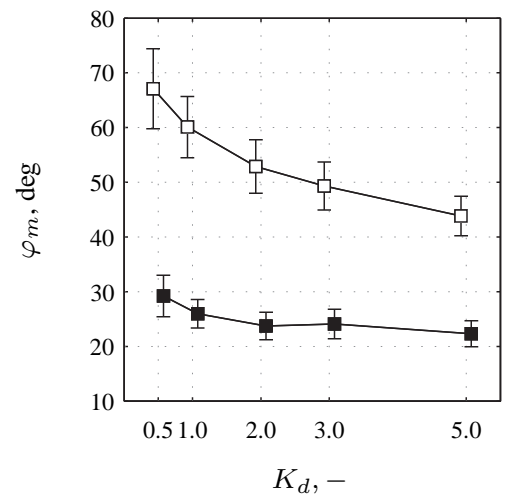

(c) Effective time delay

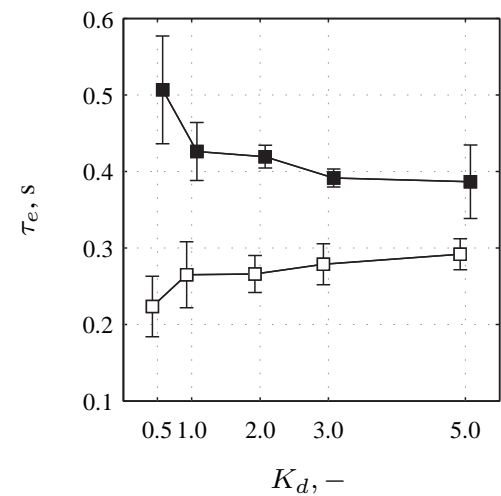

Figure 8. Crossover frequency, phase margin, and effective time delay as function of $K_{d}$.

\section{IV.B.2. Phase Margin}

In Fig. 8(b), the phase margin $\varphi_{m}$ shows a decreasing trend for increasing values of $K_{d}$ for both controlled elements, consistent with the increase in crossover frequency observed in Fig. 8(a) and the results of previous experiments. ${ }^{1,2}$ This decreasing trend in $\varphi_{m}$ was found to be highly significant for both the Citation roll and double integrator dynamics, $F(4,16)=18.26, p<0.01$ and $F(4,16)=35.30, p<0.01$, respectively.

Note also from Fig. 8(b) that the phase margin for the Citation roll dynamics was found to be over 20 deg higher than the measured phase margins for the double integrator controlled element. This is consistent with the increased inherent stability of the Citation roll dynamics, as visible from the phase of $Y_{c, c i t}$ depicted in in Fig. 6, which is clearly 20-40 deg above -180 deg in the crossover region. Fig. 8(b) further shows that the decrease in phase margin was markedly larger for the Citation roll dynamics, suggesting an interaction between displayed error scaling and system dynamics. As can be verified from Table 2, this interaction was indeed found to be highly significant, $F(4,32)=$ $108.66, p<0.01$. This more pronounced effect of $K_{d}$ for the Citation roll dynamics indicates that, despite the highly similar trends in crossover frequency for both dynamics (see Fig. 8(a)), operators adapted their control behavior to the variation in $K_{d}$ in a different manner for both $Y_{c}$.

\section{IV.B.3. Effective Time Delay}

Fig. 8(c) depicts the effective time delay, calculated from measured $\omega_{c}$ and $\varphi_{m}$ according to the Crossover Model. ${ }^{2}$ The data presented in this figure show markedly opposite trends in $\tau_{e}$ for both controlled elements. The effective time delay was found to decrease with increasing $K_{d}$ for the double integrator dynamics, which is highly consistent with the higher $\omega_{c}$ and reduced $\varphi_{m}$ depicted in Figs. 8(a) and (b), respectively. ${ }^{1}$ This decrease in $\tau_{e}$ was found to be a highly significant effect for $Y_{c, d i}, F(4,16)=7.91, p<0.01$.

In contrast, Fig. 8(c) shows that, despite similarly increasing crossover frequencies and decreasing phase margins, the effective time delay is found to increase for the Citation roll dynamics. This increase in $\tau_{e}$ with increasing $K_{d}$ was also found to be a significant effect, $F(4,16)=3.97, p<0.05$. This increase in $\tau_{e}$ is believed to be a result of the dynamics of $Y_{c, c i t}$ and specifically the location of the break frequency $\omega_{b}$, that is $2.153 \mathrm{rad} / \mathrm{s}$. Note from Fig. 8(a) that $\omega_{b}$ is in the range of crossover frequencies measured for the Citation roll dynamics. Around this break frequency, the Citation roll dynamics smoothly change from $K / s$ to $K / s^{2}$, which implies that for $\omega_{c}>\omega_{b}$ pilot lead needs to be 
generated to achieve $K / s$ open-loop dynamics in the crossover region. As can be verified from Fig. 8(a), measured crossover frequencies were found to on average be higher than $\omega_{b}$ for $K_{d} \geq 2.0$. McRuer and Jex ${ }^{2}$ also report an increase in $\tau_{e}$ if the order of the controlled element dynamics in the crossover region increases from $\mathrm{K} / \mathrm{s}$ to $\mathrm{K} / \mathrm{s}^{2}$. As expected from the opposite trends in $\tau_{e}$ for both controlled elements, the interaction between $K_{d}$ and $Y_{c}$ was found to be highly significant, $F(4,32)=11.80, p<0.01$.

(a) Citation roll dynamics

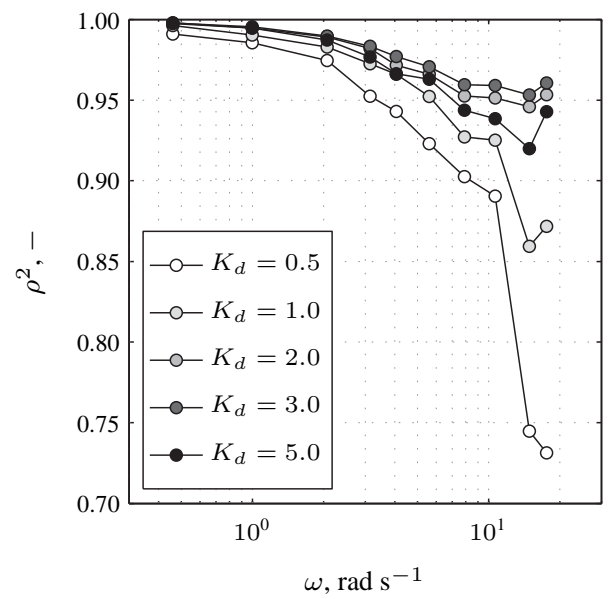

(b) double integrator

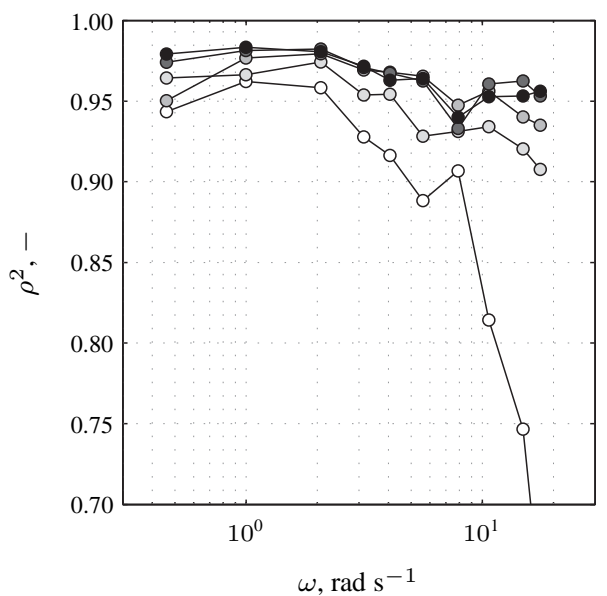

Figure 9. Average correlation coefficient as function of $K_{d}$ for both controlled elements.

\section{IV.C. Analysis of Pilot Linearity}

The $\rho^{2}$ correlation coefficient ${ }^{1}$ is analyzed to assess the linearity of pilot control behavior, that is, the correlation of the measured control inputs with the target forcing function signal $f_{t}$. The $\rho^{2}$ correlation coefficient is defined as:

$$
\rho^{2}=1-\frac{S_{n n}(j \omega)}{S_{u u}(j \omega)}
$$

This coefficient was computed at all frequencies at which $f_{t}$ had power (see Table 1). The remnant spectrum at these forcing function frequencies, $S_{n n}(j \omega)$, was calculated by averaging the noise level over the neighboring frequencies in the spectrum of $u$. the forcing frequencies for each run, after which it was averaged for every run and subject. Fig. 9 depicts the correlation coefficient, averaged over all participants and the five repetitions performed by each participant, for both controlled elements and all values of the display scaling gain $K_{d}$. Fig. 9 shows that $\rho^{2}$ was found to be well above 0.90 below the crossover frequency, indicating that the linear portion of the control actions dominated nonlinear contributions.

When looking at the effect of displayed error scaling on the correlation coefficient, the smallest display scaling gain of 0.5 shows considerably lower $\rho^{2}$ coefficients than the conditions with higher values for $K_{d}$, for both controlled elements. This suggests that at the lowest scaling level, subjects were not able to sufficiently correlate their control inputs with the tracking errors induced by $f_{t}$, which most likely resulted from the difficulty in perceiving $e$ from the compensatory display. Results show a considerable improvement for display scaling gains of 1.0 and 2.0, which seems to level of for even higher values of $K_{d}$. As can be verified from Fig. 9, no marked differences in $\rho^{2}$ were observed for the three highest considered values of $K_{d}$, especially for the double integrator dynamics (Fig. 9(b)). Overall, Fig. 9 shows a marked effect of $K_{d}$ on the correlation of control inputs with the target forcing function signal, which is in line with the first hypothesis of the experiment.

\section{IV.D. Pilot Modeling Results}

For analysis of the adopted pilot control strategy, the Precision Model (see Section II.B) was fit to the measured time traces of $e$ and $u$ using a time-domain maximum likelihood estimation procedure. ${ }^{20}$ Note that for both the double integrator and Citation roll dynamics, pilots would only need to provide lead equalization for achieving $\mathrm{K} / \mathrm{s}$ dynamics around crossover. Therefore, the lag term present in Eq. (2) was not considered in the pilot model for both types of dynamics. As can be verified from Fig. 10 and 11, which show example pilot modeling results for double integrator and 
(a) Magnitude

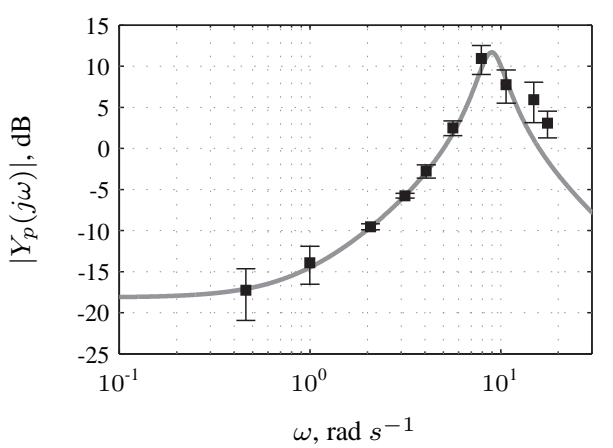

(b) Phase

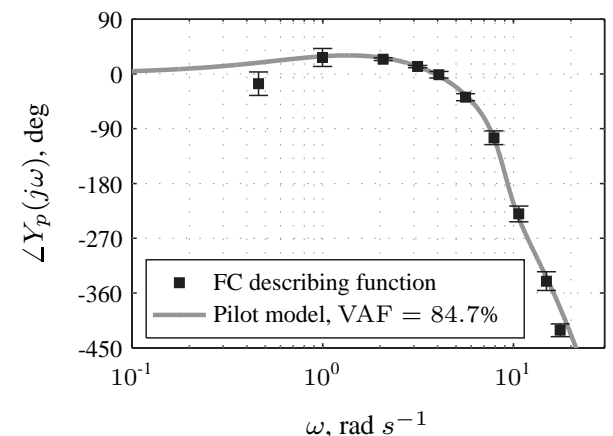

Figure 10. Example pilot model estimate with corresponding Fourier coefficients (subject 1, double integrator dynamics, $K_{d}=1.0$ ).

(a) Magnitude

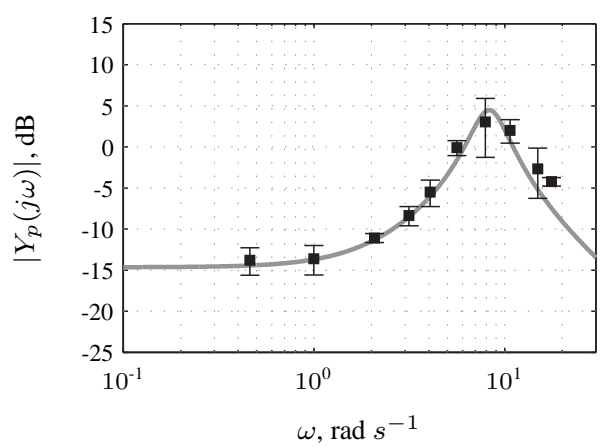

(b) Phase

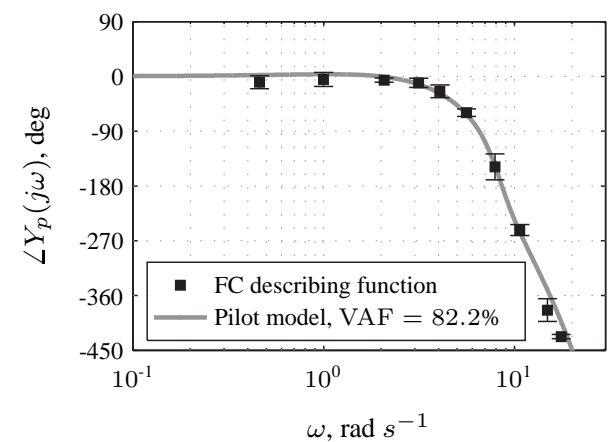

Figure 11. Example pilot model estimate with corresponding Fourier coefficients (subject 2, Citation roll dynamics, $\left.K_{d}=1.0\right)$.

Citation roll dynamics, respectively, pilot describing function estimates obtained using the Fourier coefficients (FC) method $^{27}$ confirm the absence of pilot lag equalization. The remainder of this section describes the further analysis of the pilot model parameter estimates that correspond to the model responses shown in Figs. 10 and 11, which are shown in Figs. 12 and 13. Both these graphs depict the average estimated parameters and variance bars that indicate the $95 \%$ confidence intervals, similar to Fig. 8.

\section{IV.D.1. Pilot Gain}

Fig. 12(a) shows that for both controlled elements increased $K_{d}$ also yielded an increase in the pilot gain $K_{p}$. In addition, this increase in $K_{p}$ was found to be highly significant for both the Citation roll and double integrator dynamics: $F(4,16)=32.67, p<0.01$ and $F(4,16)=10.95, p<0.01$, respectively. The increasing trend in pilot gain shown in Fig. 12(a) is in agreement with the increased tracking performance and crossover frequencies depicted in Sections IV.A and IV.B. These results further confirm that the enhanced perception of tracking errors for higher values of $K_{d}$ allowed for more accurate control, with a higher gain.

As can be verified from Fig. 12(a), the increase in $K_{p}$ is largest for the lower levels of error scaling, similar to what was found for $\omega_{c}$ (Fig. 8). However, the increase in pilot gain is markedly larger for the Citation roll dynamics, suggesting an interaction between displayed error scaling and controlled element dynamics. The mixed-design ANOVA results (Table 2) confirmed the presence of a highly significant interaction between displayed error scaling and the dynamics of $Y_{c}, F(4,32)=10.31, p<0.01$.

\section{IV.D.2. Lead Time Constant}

As can be seen in Fig. 12Fig. 12b, the lead time constant was found to steadily decrease with increasing $K_{d}$. Again, the main effect of $K_{d}$ on $\tau_{L}$ was found to be highly significant for both the Citation roll and double integrator controlled elements $(F(4,16)=9.94, p<0.01$ and $F(4,16)=5.67, p<0.01$, respectively). Note that as the inverse of the lead time constant indicates the frequency from which pilot lead was generated, a reduction in $\tau_{L}$ signals a decrease in the amount of lead equalization adopted during manual control. Fig. 12(b) shows that the lead time constant was 
(a) Pilot gain

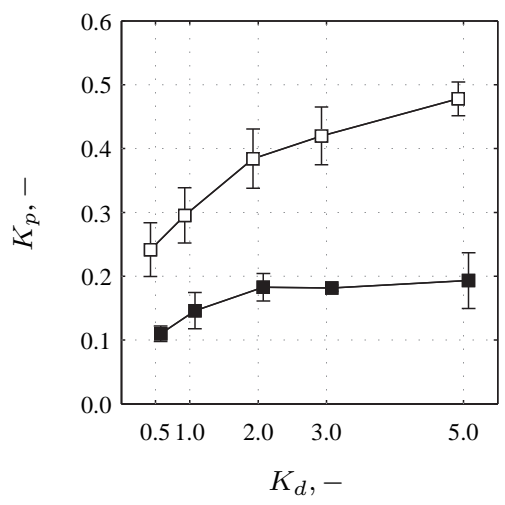

(b) Lead time constant

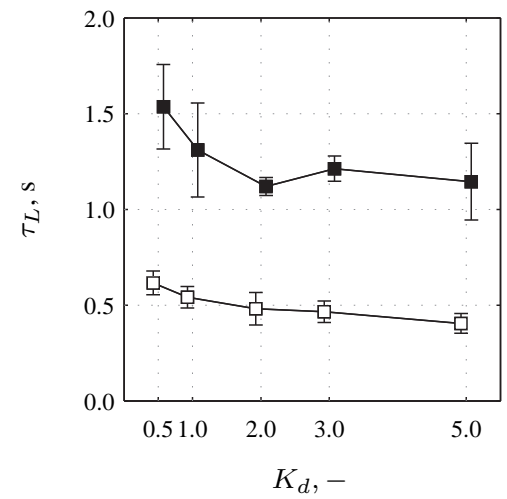

(c) Pilot time delay

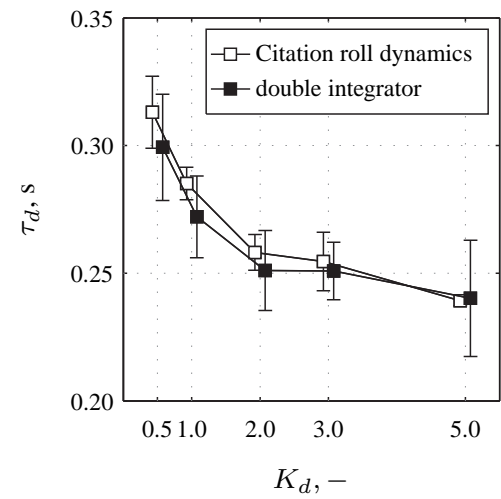

Figure 12. Pilot gain, lead time constant and pilot time delay as function of $K_{d}$.

found to be clearly higher for control of the double integrator dynamics, which was already predicted based on the comparison of both dynamics in Section III.A.3 and the work of McRuer and Jex. ${ }^{2}$

Furthermore, note that the lead time constant for Citation roll dynamics was approximately equal to the inverse of the break frequency, $1 / \omega_{b}=0.464 \mathrm{~s}$, which is expected based on the results of McRuer and Jex. ${ }^{1}$ For low values of $K_{d}$ lead constants are found to, on average, be higher than $1 / \omega_{b}$, indicating that more lead is generated than is strictly required to achieve $K / s$ open-loop dynamics around crossover.

The observed trends in $\tau_{L}$ are highly similar for both controlled elements, except for some deviations in the double integrator data for $K_{d}=3.0$, where the results for the double integrator dynamics show a slight increase in $\tau_{L}$. Still, the mixed-design ANOVA confirmed that these trends were not significantly different, $F(4,32)=1.81, p>0.05$.

\section{IV.D.3. Pilot Time Delay}

Fig. 12(c) shows that the pilot time delay was found to markedly decrease for increasing values of the display scaling gain $K_{d}$. Note that compared to the condition with $K_{d}=0.5$, pilot time delays were measured to be over $0.05 \mathrm{~s}$ lower for the higher display scaling gains. This decrease in $\tau_{d}$ indicates that for higher values of $K_{d}$ participants were able to react to presented tracking errors with reduced latency.

Furthermore, note that this decreasing trend in $\tau_{d}$ is nearly identical and highly statistically significant for both controlled elements (Citation roll dynamics: $F(4,16)=61.96, p<0.01$; double integrator dynamics: $F(4,16)=$ 10.81, $p<0.01)$. As can be verified from Table 2 no interaction between $K_{d}$ and $Y_{c}$ was found for $\tau_{d}$, as expected when considering Fig. 12(c).

\section{IV.D.4. Neuromuscular Bandwidth}

Fig. 13 depicts the identified parameters of the neuromuscular system model, see Eq. (2). As can be verified from Fig. 13(a), an increase in displayed error scaling is found to yield an increase in the bandwidth of the neuromuscular system model of around $1.5 \mathrm{rad} / \mathrm{s}$, for both controlled elements. Despite the comparatively large confidence intervals depicted in Fig. 13(a), this effect of $K_{d}$ on the neuromuscular bandwidth was found to be highly significant for both the Citation roll and double integrator controlled elements: $F(4,16)=11.50, p<0.01$ and $F(4,16)=20.99, p<0.01$, respectively. As expected, no significant interaction between displayed error scaling and system dynamics was found, $F(4,32)=0.97, p>0.05$. This is supported by Fig. 13a, which shows the curves for both system dynamics to be nearly parallel. Similar increases in $\omega_{n m}$ have been reported for previous experiments, for instance due to the addition of motion feedback. ${ }^{8,9}$ This increase in $\omega_{n m}$ can be explained by an increase in the intrinsic stiffness of the subject's arm, potentially due to increased co-contraction, ${ }^{21}$ as a result of subjects' adopting a tighter grip on the stick when controlling with a higher pilot gain (see Fig. 12(a)).

\section{IV.D.5. Neuromuscular Damping Coefficient}

The identified for the neuromuscular damping coefficient depicted in Fig. 13(b) suggest that both the variations in $K_{d}$ and controlled element dynamics had very little effect on $\zeta_{n m}$, which was found to be around 0.15 for all conditions of 
(a) Neuromuscular bandwidth

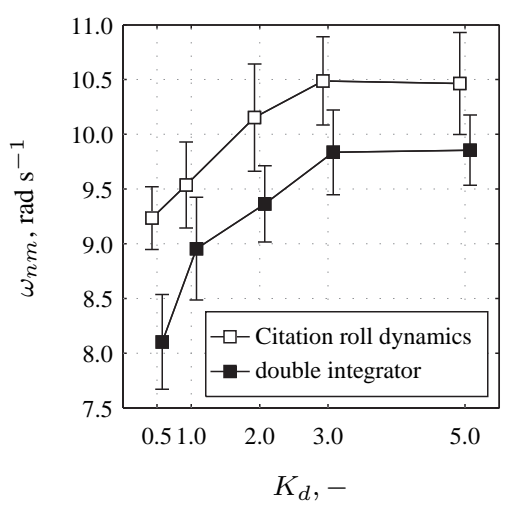

(b) Neuromuscular damping

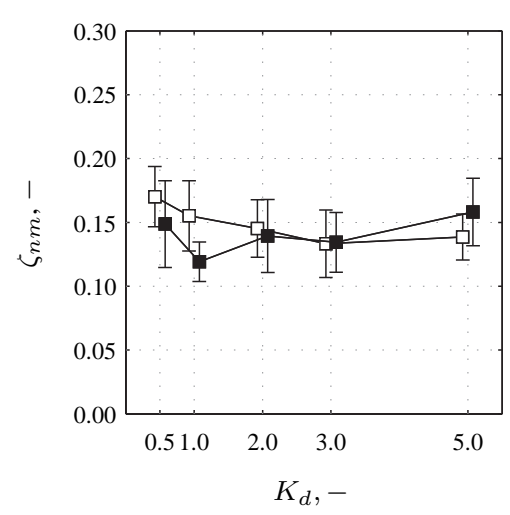

Figure 13. Neuromuscular system model parameters as function of $K_{d}$.

the experiment. This is confirmed by the ANOVA results, as can be verified from Table 2, which reveal no significant main effects of $K_{d}$ and no significant interaction with $Y_{c}$.

\section{Discussion}

The objective of the experiment described in this paper was to investigate the effects of the scaling of presented tracking errors on manual control behavior during compensatory roll-axis target-following. A compensatory display resembling an artificial horizon was used to present the roll tracking error, where the rotation of the horizon line with respect to a fixed aircraft symbol indicated the instantaneous value of the tracking error. In addition to a variation in the displayed error gain, the experiment also considered manual control for two different controlled elements: one being typical the aileron-to-roll dynamics of a small jet aircraft, the other a pure double integrator.

As the presented results show, the scaling of displayed tracking errors has a marked effect on tracking performance, the linearity of pilot control inputs, and the adopted control strategy for such a display. Markedly reduced tracking performance, $\rho^{2}$ correlation coefficients, and pilot-vehicle system crossover frequencies were observed for the lowest values of the display scaling gain $K_{d}$, including the 1-to-1 presentation of tracking errors $\left(K_{d}=1.0\right)$. For the conditions with higher display scaling gains, most considered dependent measures were found to level-off for $K_{d} \geq$ 2.0, even though analysis with McRuer et al.'s Precision Model revealed continuing adaptation of manual control behavior even for these conditions. Overall, it can be concluded that for the combination of the compensatory display and typical quasi-random forcing function signal that were considered in this experiment, a displayed error scaling gain of at least 2.0 is found to be required to ensure manual control behavior is not negatively affected by difficulties in perceiving $e$.

Due to the significantly reduced stability of the double integrator dynamics, and hence the more difficult control task, it was anticipated that the effects of scaling of presented tracking errors would be larger for this controlled element than for the Citation roll dynamics. The improvement in tracking performance with increased display gain was indeed found to be more pronounced for the double integrator dynamics. For most other considered dependent measures, however, mixed ANOVA analysis revealed no significant interactions between the variation in display gain and controlled element dynamics, implying highly similar effects of displayed error scaling for both dynamics. Measured phase margins and pilot gains were even found to be more significantly affected by the displayed error scaling for the Citation roll dynamics than for the double integrator controlled element. Therefore, the conclusion must be drawn that even though performance measures indicated a larger effect of $K_{d}$ for the double integrator dynamics, this is not reflected in the underlying control behavior, for which measured changes resulting from displayed error scaling were found to be largely invariant with controlled element dynamics.

Though not officially part of the experiment, during debriefing participants were asked to indicate (if possible) which setting of the display gain they preferred. None of the participants indicated a preference for a display scaling gain of 5.0. All subjects except one identified gain values of either 2.0 or 3.0 as their preferred setting and several specifically commented on the $K_{d}=5.0$ condition, stating that they experienced the presented tracking errors as overly scaled-up. It would be of much interest to the experiment described in this paper to also explicitly take such subjective measures into account, and investigate possible correlation with the objective measures analyzed here, in further experiments on the effect of displayed error scaling on manual control during tracking. 
For some of the considered dependent measures, the results presented in this paper indicate marked differences for the two controlled elements that were considered in the experiment. An example of this is that crossover frequencies were found to be around $0.5 \mathrm{rad} / \mathrm{s}$ lower for the Citation roll dynamics than for the double integrator. Based on the higher stability of the Citation roll dynamics, this is an unexpected result, as previous work ${ }^{1,2}$ suggests control with a higher crossover frequency would be adopted for such controlled element dynamics than for double integrator control. Drawing definite conclusions on these effects of the controlled element based on the presented data is difficult due to the fact that the experiment had a mixed design in which the different controlled elements were not considered as a within-subjects factor. To further investigate the reported interactions between controlled element dynamics and displayed error scaling, it is preferable to perform an experiment in which all participants also control both system dynamics.

\section{Conclusions}

An experiment was performed to investigate the effects of displayed tracking error scaling on manual control behavior during a compensatory roll attitude target-following task. For a compensatory display that presented roll tracking errors as a rotation with respect to fixed reference, increased tracking performance and linearity of manual control behavior were anticipated for higher display scaling gains. The results indeed indicated increases in tracking performance, crossover frequency, pilot gain and neuromuscular bandwidth with increased display scaling. In addition, pilot time delays, the amount of visual lead equalization performed by participants during tracking, and measured pilot-vehicle system phase margins were found to decrease for higher display gains. Analysis of the $\rho^{2}$ correlation coefficient revealed markedly decreased correlation of control inputs for display scaling gains of 0.5 and 1.0 , indicating tracking errors need to be scaled by at least 2.0 for the considered compensatory display to avoid artifacts in measured manual control behavior. Furthermore, most of the observed effects of displayed error scaling were found to be largely invariant with the different controlled element dynamics considered in the experiment.

\section{References}

\footnotetext{
${ }^{1}$ McRuer, D. T., Graham, D., Krendel, E. S., and Reisener, W., "Human Pilot Dynamics in Compensatory Systems. Theory, Models and Experiments With Controlled Element and Forcing Function Variations," Tech. Rep. AFFDL-TR-65-15, 1965.

${ }^{2}$ McRuer, D. T. and Jex, H. R., "A Review of Quasi-Linear Pilot Models," IEEE Transactions on Human Factors in Electronics, Vol. HFE-8, No. 3, 1967, pp. 231-249.

${ }^{3}$ Wasicko, R. J., McRuer, D. T., and Magdaleno, R. E., "Human Pilot Dynamic Response in Single-Loop Systems with Compensatory and Pursuit Displays," Tech. Rep. AFFDL-TR-66-137, 1966.

${ }^{4}$ Stapleford, R. L., Peters, R. A., and Alex, F. R., "Experiments and a Model for Pilot Dynamics with Visual and Motion Inputs," Tech. Rep. NASA CR-1325, 1969.

${ }^{5}$ Jex, H. R., Magdaleno, R. E., and Junker, A. M., "Roll Tracking Effects of G-vector Tilt and Various Types of Motion Washout,” Fourteenth Annual Conference on Manual Control, 1978, pp. 463-502.

${ }^{6}$ Van der Vaart, J. C., Modelling of Perception and Action in Compensatory Manual Control Tasks, Ph.D. thesis, Faculty of Aerospace Engineering, Delft University of Technology, 1992.

${ }^{7}$ Hosman, R. J. A. W., Pilot's perception and control of aircraft motions, Ph.D. thesis, Faculty of Aerospace Engineering, Delft University of Technology, 1996.

${ }^{8}$ Zaal, P. M. T., Pool, D. M., De Bruin, J., Mulder, M., and Van Paassen, M. M., "Use of Pitch and Heave Motion Cues in a Pitch Control Task," Journal of Guidance, Control and Dynamics, Vol. 32, No. 2, 2009, pp. 366-377.

${ }^{9}$ Pool, D. M., Zaal, P. M. T., Mulder, M., and Van Paassen, M. M., "Effects of Heave Washout Settings in Aircraft Pitch Disturbance Rejection," Journal of Guidance, Control, and Dynamics, Vol. 33, No. 1, 2010, pp. 29-41.

${ }^{10}$ Hess, R. A. and Malsbury, T., "Closed-loop Assessment of Flight Simulator Fidelity," Journal of Guidance, Control, and Dynamics, Vol. 14, No. 1, 1991, pp. 191-197.

${ }^{11}$ Steurs, M., Mulder, M., and Van Paassen, M. M., "A cybernetic approach to assess flight simulator fidelity," Proceedings of the AIAA Modeling and Simulation Technologies Conference and Exhibit, Providence, Rhode Island, 16-19 August 2004, 2004, AIAA-2004-5442.

${ }^{12}$ Zaal, P. M. T., Pool, D. M., Mulder, M., Van Paassen, M. M., and Mulder, J. A., "Identification of Multimodal Pilot Control Behavior in Real Flight," Journal of Guidance, Control and Dynamics, Accepted for publication.

${ }^{13}$ Hess, R. A., "A model-based analysis of handling qualities and adverse aircraft-pilot coupling in high angle of attack flight," Proceedings of the 1995 IEEE International Conference on Systems, Man \& Cybernetics, Vancouver, Canada, 1995, pp. 2663-2669.

${ }^{14}$ Damveld, H. J., A Cybernetic Approach to Assess the Longitudinal Handling Qualities of Aeroelastic Aircraft, Ph.D. thesis, Faculty of Aerospace Engineering, Delft University of Technology, 2009.

${ }^{15}$ Pool, D. M., Mulder, M., Van Paassen, M. M., and Van der Vaart, J. C., "Effects of Peripheral Visual and Physical Motion Cues in Roll-Axis Tracking Tasks," Journal of Guidance, Control, and Dynamics, Vol. 31, No. 6, 2008, pp. 1608-1622.

${ }^{16}$ Hess, R., "Modelling the Effects of Display Quality upon Human Pilot Dynamics and Perceived Vehicle Handling Qualities," IEEE Transactions of Systems, Man, and Cybernetics, Vol. 25, 1995, pp. 338-344.
} 
${ }^{17}$ Beerens, G. C., Damveld, H. J., Mulder, M., Van Paassen, M. M., and Van der Vaart, J. C., "Investigation into Crossover Regression in Compensatory Manual Tracking Tasks," Journal of Guidance, Control, and Dynamics, Vol. 32, No. 5, 2009 , pp. 1429-1445.

${ }^{18}$ Pool, D. M., Zaal, P. M. T., Van Paassen, M. M., and Mulder, M., "Identification of Roll Attitude Control Behavior During Turn Maneuvers," Proceedings of the AIAA Modeling and Simulation Technologies Conference and Exhibit, Chicago (IL), No. AIAA-2009-6029, 2009.

${ }^{19}$ Zaal, P. M. T., Pool, D. M., Mulder, M., and Van Paassen, M. M., "New Types of Target Inputs for Multi-Modal Pilot Model Identification," Proceedings of the AIAA Modeling and Simulation Technologies Conference and Exhibit, Honolulu (HI), No. AIAA-2008-7106, 2008.

${ }^{20}$ Zaal, P. M. T., Pool, D. M., Chu, Q. P., Van Paassen, M. M., Mulder, M., and Mulder, J. A., "Modeling Human Multimodal Perception and Control Using Genetic Maximum Likelihood Estimation,” Journal of Guidance, Control, and Dynamics, Vol. 32, No. 4, 2009 , pp. $1089-1099$.

${ }^{21}$ Damveld, H. J., Abbink, D. A., Mulder, M., Van Paassen, M. M., van der Helm, F. C. T., and Hosman, R. J. A. W., "Measuring the Contribution of the Neuromuscular System during a Pitch Control Task," Proceedings of the AIAA Modeling and Simulation Technologies Conference and Exhibit, Chicago (IL), No. AIAA-2009-5824, 2009.

${ }^{22}$ Sadoff, M., "Effects of High Sustained Acceleration on Pilot Performance and Dynamic Response," Tech. Rep. NASA-TN-D-2067, 1964.

${ }^{23}$ Sadoff, M. and Dolkas, C. B., "Acceleration Stress Effects on Pilot Performance and Dynamic Response," 2nd Annual NASA-University Conference on Manual Control, No. NASA SP-128, 1966, pp. 241 - 258.

${ }^{24}$ Weisz, A. Z., Allen, R. W., and Goddard, C. J., “An Evaluation of Three Types of Hand Controllers Under Random Vertical Vibration,” 2nd Annual NASA-University Conference on Manual Control, No. NASA SP-128, 1966, pp. 269 - 278.

${ }^{25}$ Jex, H. R., Allen, R. W., and Magdaleno, R., "Effects of Display Format on Pilot Describing Function and Remnant," Seventh Annual Conference on Manual Control, No. NASA SP-281, NASA, Washington, DC, 1972, pp. 155-159.

${ }^{26}$ Stroosma, O., Van Paassen, M. M., Mulder, M., and Postema, F. N., "Measuring Time Delays in Simulator Displays," Proceedings of the AIAA Modelling and Simulation Technologies Conference and Exhibit, Hilton Head (SC), No. AIAA-2007-6562, 2007.

${ }^{27}$ Van Paassen, M. M. and Mulder, M., "Identification of Human Operator Control Behaviour in Multiple-Loop Tracking Tasks," Proceedings of the Seventh IFAC/IFIP/IFORS/IEA Symposium on Analysis, Design and Evaluation of Man-Machine Systems, Kyoto Japan, Pergamon, 1998, pp. 515-520. 